\title{
Almost Disjunct Codes in Large Scale Multihop Wireless Network Media Access Control
}

\author{
D. Charles Engelhart \\ Anand Sivasubramaniam \\ Penn. State University \\ University Park PA 16802 \\ engelhar, anand \\ @cse.psu.edu
}

\begin{abstract}
We discuss the application of coding constructs to the problem of radio transmission scheduling in ad hoc wireless networks. We are particularly interested in the ability to scale such ad hoc wireless networks to thousands of nodes. Current media access protocols do not scale well to larger networks than a few dozen nodes.

We examine the possible ways to apply superimposed codes and almost disjunct codes to these networks media access layer. We analyzed how well one can build codes that are not as powerful as superimposed codes but have better parameters for the case of ad hoc networks. We look at the local network performance that a particular node can expect using these codes. We also examine the system wide performance using an approximate simulation of thousands of ad hoc wireless nodes.
\end{abstract}

\section{Introduction}

We would like to improve the performance of media access control (MAC) in wireless ad hoc networks. We are interested in performance both in terms of quality of service measures like throughput and latency but also in terms of measures that will affect battery life, such as collision rate.

In order to transmit data a wireless node needs to be sure that no other nodes in its radio range are transmitting at the same time so the transmission can be heard by its neighbors.

Traditionally MAC protocols can be classified as either centralized or distributed algorithms. Centralized protocols like TDMA suffer from low spatial utilization as generally only one node in the system is allowed to transmit at a time, but guarantee no conflicts. Distributed algorithms offer the possibility of spatial reuse but suffer from significant contention problems even in moderately loaded systems, and usually cannot guarantee reliable delivery.

We are particularly interested in how these systems will scale to thousands of nodes or more. Since these nodes are usually mobile devices and thus are resource constrained, especially with regard to battery life, we will be more interested in the efficiency of the system than throughput or latency.

Recently advanced protocols based on the ideas of TDMA, reliable delivery and little contention, have started to appear. These new protocols use advanced coding structures to reuse the space and still guarantee reliable delivery.

These systems were first discussed in [2] and refined in [3]. Both protocols use constructions of orthogonal codes to ensure that a conflict free schedule can be obtained without knowledge of the underlying network topology. Both studies analyze the theoretical throughput of these systems and the latter more closely analyzes guaranteed throughput as well as scheduling delays. A recent paper by Syrotiuk, et al. [6] generalizes the use of orthogonal arrays for these systems and analyzes both the probability of successful transmission and throughput of such systems built from general orthogonal arrays of strength 2 and 3 .

The remainder of this paper is organized as follows. Section 2 goes over some basic coding constructs. The ways they can be applied to wireless networks is discussed in Section 3. Section 4 examines the localized network performance when using superimposed codes. An approximate simulation of a large scale wireless network is used to compare an almost disjunct code to csma/ca and a hybrid protocol in Section 5.

\section{Orthogonal arrays and Superimposed codes}

In general we are going to use a binary matrix to represent each nodes transmission schedule. The rows of the ma- 
trix correspond to the given time-slots that nodes can transmit in. Each column of the matrix represents a single node. Thus each time a 1 appears in a row $i$ column $j$ then node $j$ is allowed to transmit during time-slot $i$. The schedule is then repeated indefinitely. The number of columns of such a matrix will limit the number of nodes in a system. In general we will let $n$ be the number of nodes in the system.

\subsection{Orthogonal arrays}

Previous work [6] has used orthogonal arrays to construct these schedules.

Def. $O A(t, k, v)$ is a $k \times v^{t}$ matrix using the symbols $\{0,1,2 \ldots v-1\}$ is a strength $t$ orthogonal array if for any set of $t$ of its rows each $t$-tuple of column elements appears exactly once, for that row set.

One important property of such an orthogonal array is that each column, or codeword, of $O A(t, k, v)$ intersects every other column in fewer than $t$ positions. Using this property we can build a transmission schedule. First no two columns intersect in more than $t-1$ positions, so given a set of $D$ other columns the given column differs from them in at least $k-D(t-1)$ positions. As long as this difference is positive we can say that there is at least one row where the given column is different from all of any set of $D$ other columns.

If we assume this $D$ is bound for the number of neighbors any given node can have we can then assure each node has a time-slot in which it is allowed to talk when none of its neighbors are. The symbols $\{0,1,2 \ldots v-1\}$ are mapped to the set of identity vectors. Using this binary vectorization we can see that as long as 2 symbols are different then during that row the 2 nodes will not interfere with each other. So using $O A(t, k, v)$ we can handle up to $v^{t}$ nodes and a schedule includes $k * v$ time-slots.

\subsection{Superimposed codes}

We can also construct viable schedules using superimposed codes [4]. We say one binary vector $\bar{a}$ covers another $\bar{b}$ iff for every 1 in $\bar{b}$ there is a 1 in the same position in $\bar{a}$.

Def. A binary matrix is $d$-disjunct iff it has the property that the union of any $d$ codewords does not cover any codeword not in that set of $d$ codewords.

We can then construct a schedule using such a $d$-disjunct superimposed code, because we know there is at least one row where there exists a 1 corresponding to a given node and there are all 0's for all of its $d$ neighbors. Each node is guaranteed a time-slot where it can talk and none of its neighbors may.

We can then see that what we constructed with the binary vectorized orthogonal arrays was a $D$-disjunct superimposed code.

\subsection{Almost disjunct codes}

We would like to relax the property of having an absolute guarantee that there exists a time-slot in which every node is allowed to talk with no interference. To do so we wish to consider $\alpha$-almost $d$-disjunct codes [1].

Def. A binary matrix is $\alpha$-almost $d$-disjunct if for any randomly selected set of $d$ columns, the probability that they cover no other column is at least $\alpha$.

So we can say that a 1 -almost $d$-disjunct matrix is exactly a $d$-disjunct superimposed code.

These matrices have the property that each node has probability $\alpha$ that it has a time-slot where it can have interference free communication.

\subsubsection{The Matrix * Operator}

One way of increasing the number of nodes that a code can handle without increasing its schedule length much is by using the matrix * operator. The * operator applied to a single matrix produces another matrix that has every ordered pair of columns from the first matrix. Given a matrix, $M$, the matrix $M^{*}$ then has twice as many rows, but the number of columns is squared.

It is worth noting that given any matrix $M$ we cannot guarantee that $M^{*}$ is even 2-disjunct, but it may very well be highly $\alpha$-almost $d$-disjunct for a given $d$.

\section{Superimposed codes in wireless networks}

The schedules in previous work have been built on $O A(2, v, v)$ in [2] and enhanced to $O A(2, v+1, v)$ in [6] for an increase in throughput. The resulting schedules will then have $v^{2}$ codewords of length either $v^{2}$ or $v^{2}+v$ and can handle up to $v$ neighbors, that is it will be a $v$-disjunct superimposed code.

In [6] they also discuss the addition of strength 3 orthogonal codes and show that for small numbers of neighbors that they can significantly increase throughput. They again focus on orthogonal arrays where $k=v+1$, in doing so they increase the number of codewords to $v^{3}$. However, by not increasing the length of each codeword they can have a maximum of $(v+1) / 2-1$ neighbors, which roughly cuts in half the power of the superimposed code. In order to not reduce the power of the resulting superimposed code, and thus the maximum number of neighbors allowed in the system they would have to find an orthogonal array with $k$ approximately twice $v$. This would yield a schedule with codewords of length approximately $4 v^{2}$.

It can be seen that in the initial constructions using orthogonal arrays that the length of each codeword and the number of codewords is asymptotically the same. This means that a schedule must be as long as the number of 
nodes in the system. So in regard to schedule length these schedules are no better than traditional TDMA. They do, however, significantly increase the amount of data that it is possible to transmit during that time period.

If we plan to use these schedules for very large systems, they are also going to be required to have a very large schedule length, approximately the size of the number of nodes. For example in a system with one million nodes we would need to construct $O A(2,1000,1000)$ (is this easy to do?). We could then handle 1000 neighbors among any of the one million nodes, this may seem a bit too powerful for what our needs may be, perhaps we would need to only be able to handle 100 neighbors. More importantly each schedule has one million time-slots. It may be the case that certain nodes are only allowed to transmit during one small section of that schedule, because they are only guaranteed one time-slot in which none of their neighbors are allowed to transmit. This would possibly lead to large latencies, and if we want to increase the number of nodes further it could lead to near starvation.

\subsection{Incidence matrix construction of superim- posed codes}

One well known [5] construction of superimposed codes is based on the idea of incidence matrices of sets. Each row and column in such a matrix represents a given subset of $m$ objects. We then say there is a 1 in position $(i . j)$ iff the set corresponding to row $i$ is completely contained in the set corresponding to column $j$. A $d$-disjunct superimposed code can be constructed with $\left(\begin{array}{c}m \\ d\end{array}\right)$ rows (time-slots) and $\left(\begin{array}{c}m \\ s\end{array}\right)$ columns (nodes) where $m<s<d$.

Consequently we can have superimposed codes that asymptotically grow much faster in the number of codewords than the schedule length. We can see that even with a relatively small $d$ these codes are only going to be suited for large systems. The other main factor that will contribute to their performance will be the density of the codes, that is the percentage of ones that appear in the code, that is the percentage of time the nodes are allowed to transmit.

\subsection{Constructing Almost disjunct codes}

Our initial method for constructing almost disjunct codes is to simply remove some percentage of the rows. By doing this we are cutting the schedule length but we are also potentially reducing the power of the code. We need to be careful that we do not remove rows in a manner which overly degrades the power of the code, or one that penalizes some nodes significantly. We will remove rows uniformly at random in order to try and minimize these cases. This is not guaranteed to minimize conflicts, but on average should penalize no codeword(node) more than any other.
With this reduction in schedule length we are not going to be able to make the same guarantees as before but hopefully these scheduling conflicts can be minimized to the point that they only occur with very low probability.

The way in which the superimposed were initially constructed will make the density of the code remain constant when removing rows.

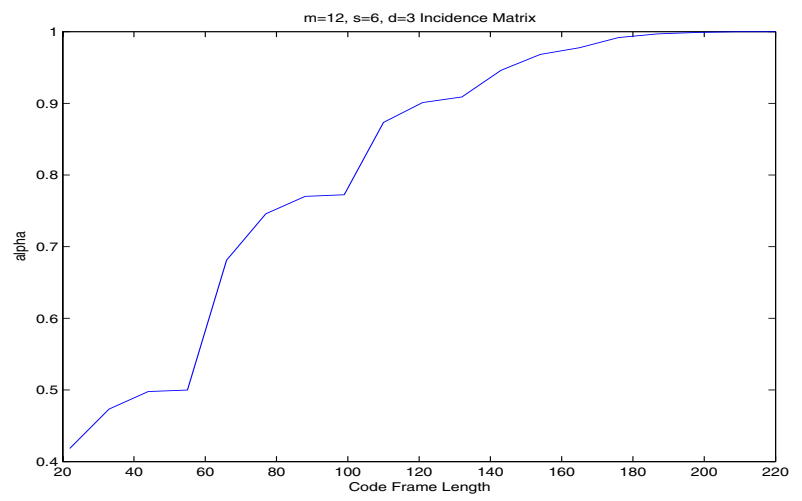

\section{Figure 1. The $\alpha$-almost disjunct property as rows are removed from the superimposed code.}

Figure 1 shows how alpha changes as we remove rows in the superimposed codes constructed with the incidence matrix construction. There were 220 rows in the initial code, notice that with only $1 / 4$ of the original rows that the code is still 0.5 -almost 3 disjunct, and with half the rows removed it is still about 0.85 -almost 3 disjunct.

We can also use a less powerful superimposed code than the number of neighbors. For example with a power 3 superimposed code we can only guarantee success in the case of 3 or less neighbors, but it is very likely that we will still have some success with more than 3 neighbors.

Figure 2 shows the alpha values in the case of a $d=3$ superimposed code with up to 30 neighbors. Using a code that is only guaranteed to be 3 disjunct it is 0.95 -almost 15 disjunct, and 0.6 -almost 30 disjunct.

It appears there are great gains to be had if we are willing to deal with a chance that our code does not strictly meet the disjunct property of a superimposed code. In a wireless network this would mean that on a given schedule there is a $1-\alpha$ chance that a node would not be guaranteed a time slot where only it was talking amongst its neighbors.

\subsection{Parameter Comparison}

Figure 3 compares the schedule length of 7-disjunct superimposed codes constructed with orthogonal arrays and the incidence matrix construction, using completely disjunct codes. The $O A(2, v, v)$ code has the longest schedule 


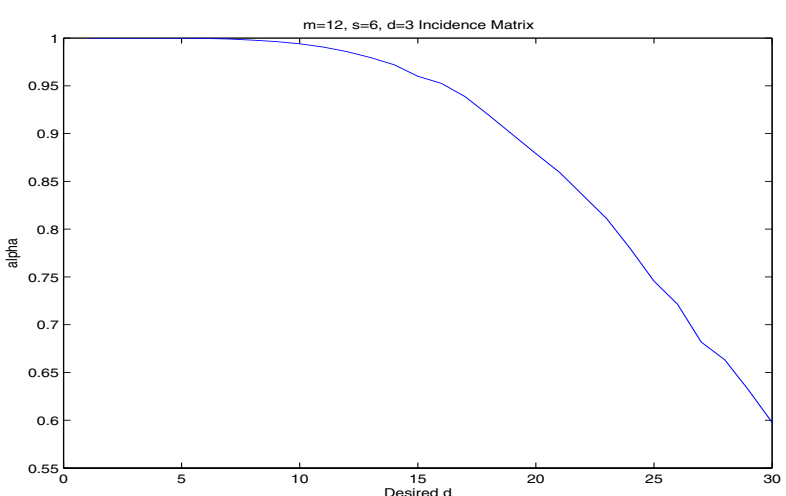

Figure 2. The $\alpha$-almost disjunct property with a lower power superimposed code.

lengths of the three over all values of network sizes. The $O A(2,8, v)$ orthogonal code performs better at lower numbers of nodes, but when the network size is increased significantly the growth rate of the incidence matrix superimposed code has shorter schedule lengths.

Table 3.3 shows the various parameter constraints for the codes discussed.

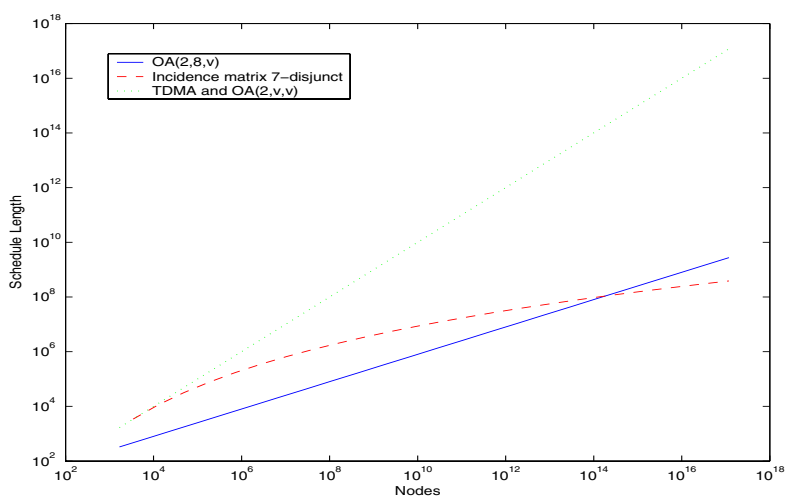

Figure 3. The schedule length of various 7disjunct superimposed codes.

\section{Localized Network Performance}

In order to understand how a wireless network will perform we will first look at the theoretical network performance in the neighborhood around a given node.

\subsection{Code Density}

For superimposed codes one of the primary characteristics that will effect network performance is the density of

\begin{tabular}{|l|l|l|l|}
\hline & nodes & schedule length & neighbors \\
\hline TDMA & $n$ & $n$ & $n$ \\
\hline orthogonal codes & $v^{t}$ & $v * k$ & $\frac{k-1}{t-1}$ \\
\hline$O A(2, v, v)$ codes & $v^{2}$ & $v^{2}$ & $v$ \\
\hline incidence matrix & $\left(\begin{array}{c}m \\
s\end{array}\right)$ & $\left(\begin{array}{l}m \\
d\end{array}\right)$ & $d$ \\
\hline almost disjunct & $\left(\begin{array}{c}m \\
s\end{array}\right)$ & $\beta *\left(\begin{array}{c}m \\
d\end{array}\right)$ & $? ?$ \\
\hline
\end{tabular}

Table 1. Parameters of various MAC protocol codes.

the code. So in order to be able to talk about how it should perform we will want expressions for the density (the fraction of ones) of each code.

The simplest way to look at the density of a superimposed code constructed via an incidence matrix is not to look at the ones, but instead the situations that will lead to zeros in the code. It is clear that a superimposed code constructed via the incidence matrix method will have the same number of ones or zeros in each column, and similarly in each row. So to find the density of the code we only need to look at one row or column.

Recall that a row in such a superimposed code corresponds to a set of $d$ objects chosen from $m$ objects, similarly columns correspond to a set of $s$ objects chosen from those same $m$ objects. Looking at any column we can see that the only time a one will appear in any position is when the set corresponding to the row is completely contained in the set corresponding to that column. There are exactly $\left(\begin{array}{l}s \\ d\end{array}\right)$ sets of size $d$ that are completely contained in the $s$-set corresponding to a given column. Since there are exactly elements in a given column the code density is then $\frac{\left(\begin{array}{l}s \\ d\end{array}\right)}{\left(\begin{array}{c}m \\ d\end{array}\right)}$. This density remains constant across rows of the matrix as well as the columns.

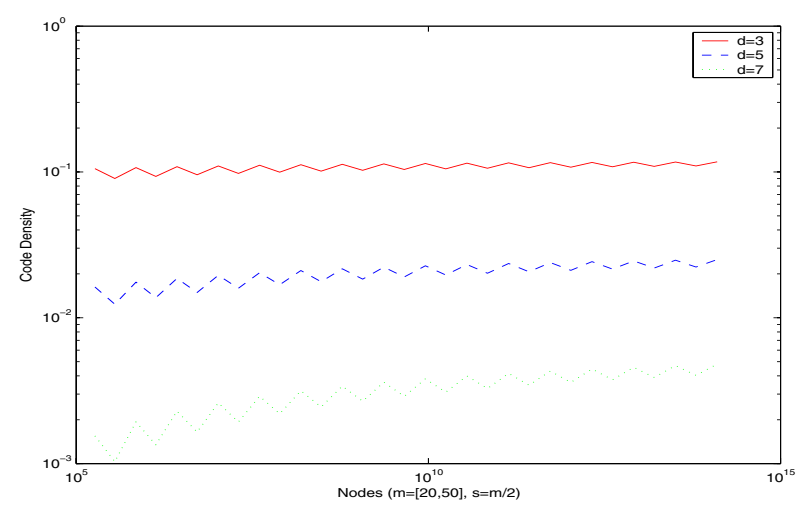

Figure 4. Code density as the number of nodes increases. 
Figure 4 shows how the density of the code changes as the number of nodes (codewords) in the system increases for 3, 5 and 7-disjunct superimposed codes, constructed with the incidence matrix construction. The values for $m$ are in the interval $[20,50]$, and the $s$ values are always chosen to be half of a given $m$, remember that $m$ and $s$ determine the maximum number of nodes for a given superimposed code. Notice that as the power of the superimposed is increased the density of the code is lower. Also as the number of nodes increases the density increases slightly.

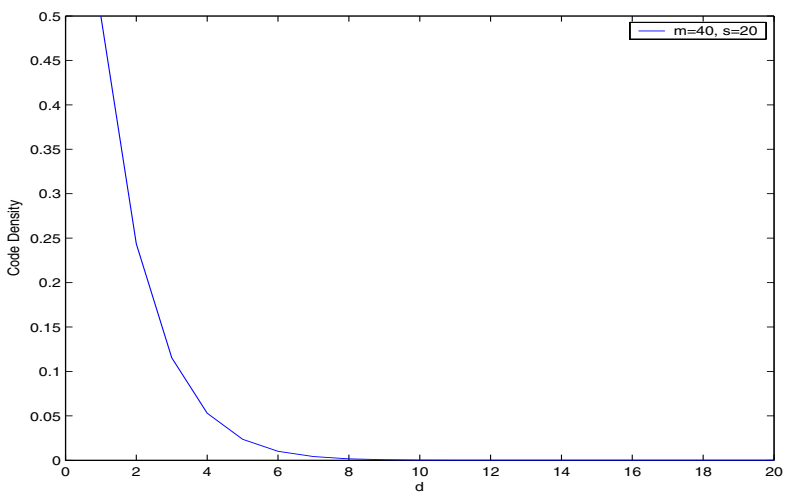

Figure 5. Code density as the power, $d$, of the superimposed code increases.

Figure 5 shows how the density changes with the power, $d$, of the superimposed code. As we saw before as the power of the code increases its density decreases.

This density remains constant as we remove rows to from an almost disjunct code.

The density for standard TDMA is clearly $1 / n$ and for orthogonal arrays is $1 / v$ which in the case of $O A(2, v, v)$ codes is $1 / \sqrt{n}$. These codes will all be somewhat inflexible if we wish to change the density of the code to suit a particular network configuration, but we have some choice in code density when using an incidence matrix superimposed code.

\subsection{Throughput}

Throughput at the MAC layer is a simple function of the reception success rate for a given node that wishes to transmit. We derive a simplified model of reception success that ignores complex propagation details.

Let $P_{r}$ be the probability of a successfully received transmission. $P_{r}$ will depend on several factors. The probability that the node is allowed to transmit during that times lot, $P_{a}$, is the first factor. Second, this success rate will also depend on the number of neighbors, $d$, that node has as well as the likelihood that a given node will wish to transmit, $P_{b}$.
A successful transmission then occurs when the node in question is allowed to transmit and all of its neighbors are silent. Thus the probability of successful reception for a node that wishes to communicate is simply $P_{r}=P_{a}(1-$ $\left.P_{a}\right)^{d * P_{b}}$

Figure 6 shows how the expected reception ratio changes as the density of the code increases, with a fixed number of neighbors. Notice that when all nodes wish to communicate all the time the desired code density is higher than in a more lightly loaded system.

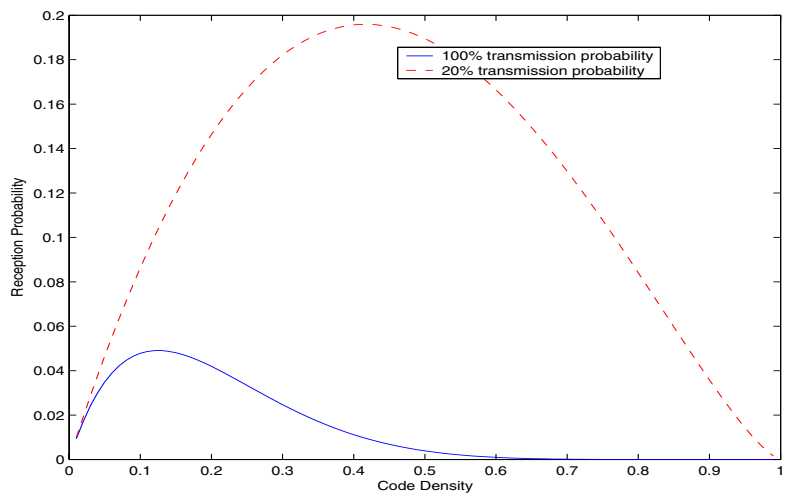

Figure 6. The reception probability as the density of the code changes.

Figure 7 shows how the reception probability changes with varying the number of neighbors, for various code densities. As expected increasing the number of neighbors reduces the probability of a successful communication, but notice how the lower density codes become more desirable as the number of nodes increases.

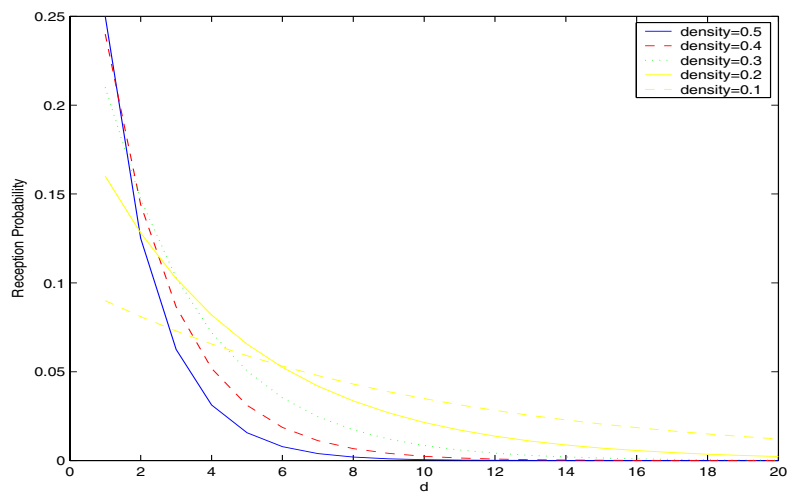

Figure 7. The reception probability as more neighbors are added to the system.

Figure 8 shows how varying both the number of neigh- 
bors and transmission probability effects the reception probability.

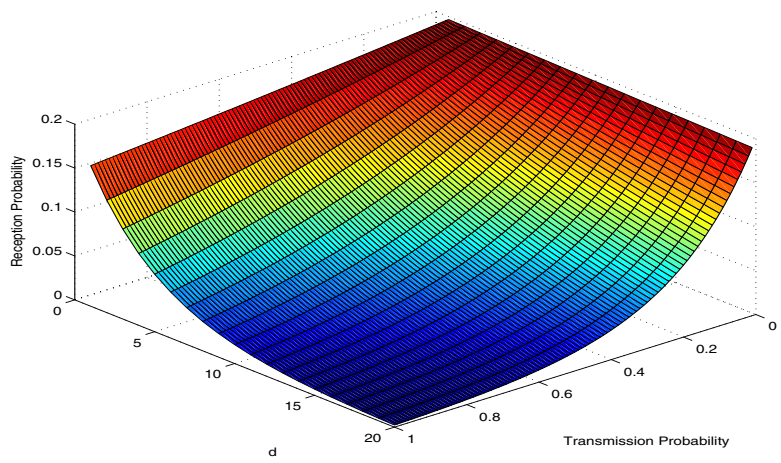

Figure 8 . The reception probability varying with both the number of neighbors and their transmission probability.

\section{Simulation}

Although looking at the performance localized around a group of nodes may give us some insight into how to optimize such networks we must look at the whole network working together in order fully analyze any MAC protocol.

To do this we use an approximate simulation that models a complete, large scale, wireless network. We used Matlab [7] to simulate these networks.

The nodes in the networks we simulated are all static, that is they do not move over the course of the simulation. They are distributed uniformly at random over a square area.

Radio propagation is handled by simply calculating the distance between pairs of nodes and if they are within some threshold distance of each other they can communicate, and otherwise they cannot.

Messages are generated using intervals determined by using an exponential distribution, governed by a parameter $\lambda$. As $\lambda$ increases the time between message generation shortens, thus there is more load in the system. Messages are sent from any random node to another random node. Routes for the messages are made using a geographic algorithm for simplicity, the next hop of a message is sent to the node in its communication range that is geographically closest to its final destination. The communication ranges were set so that there would be 16 neighbors per node on average.

We used a $1 \mathrm{mbps}$ radio model, using 512 byte packets. We ignored all packets delivered during an initial start up window, and most of the simulations ran for a few minutes of simulated time.
We ran two experiments for each radio model. First we varied the size of the network and kept the load (determined by the parameter $\lambda$ in the exponential traffic generator) constant. Second we kept the network size constant and varied the load in the system generated by the exponential traffic generator.

For each experiment we measured the total number of routing layer packets delivered, the collision rate and the average latency for routing layer packets. Note that the collision rate is going to have a direct effect on the power used by nodes in the system, as collisions are simply transmissions where power was consumed and no work was done.

\subsection{MAC Protocols}

We compare three different MAC protocols for each of the simulations.

First we used a csma/ca protocol. We used time intervals that were smaller than the time to transmit a packet ( $1 / 25$ th the time to send a packet) for radio contention. In this model a given transceiver only tries to communicate if no one is currently using the channel. If there is a collision, or anytime that the radio wants to talk after it has heard another transceiver talking it must wait a random number of the smaller time intervals before it can try to transmit. That random amount of timer intervals is based on an exponential back off timer. The back off window has a minimum size of 8 time intervals and a maximum of 256, with it doubling every time the node cannot communicate, and resetting to 8 every time it communicates successfully.

We also used a code based MAC protocol. For simplicity sake we used a very simple code that has good $\alpha$-almost disjunct properties. We used the minimum $I_{n}^{*}$ matrix that could support the number of nodes used in the simulation, for example if there were 100 simulated nodes we would have used $I_{1} 0^{*}$, which has a schedule length of 20 and a code density of 0.1 . We did not use any smaller time intervals for this simulation, and the nodes ability to communicate during a transmission window is completely based on the column of the code corresponding to that node.

In order to try to reduce the latency problems associated with the code based MAC protocol we used a hybrid approach that used the same code used for the code based protocol, but also incorporates the smaller time intervals. So a given node can start transmitting during a time slot where it has a 1 and continues transmitting until it delivers the whole packet (which takes 25 of the smaller time units). There is also a carrier sense mechanism used so that no node will try to start communicating when another node is already transmitting. 


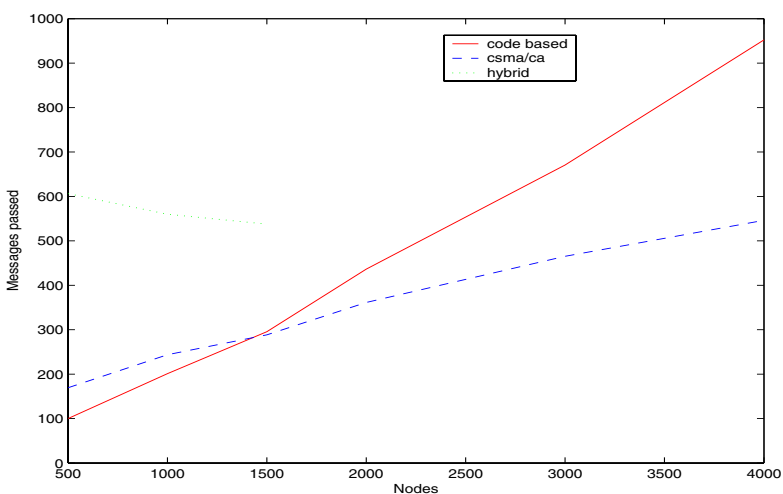

Figure 9. Routing layer messages passed as the size of the network grows.

\subsection{Network size scaling}

First we examine how each of the protocols scales as the network grows in size. The parameter for the traffic generator, $\lambda$, was kept constant at 0.0001 .

Figure 9 shows the number of messages passed for each protocol. The code based protocol starts out the worst of the 3 when there are fewer nodes in the system, but it overtook the csma/ca approach around 1500 nodes. It is worth noting the hybrid approach completely failed (near 0 throughput, 100

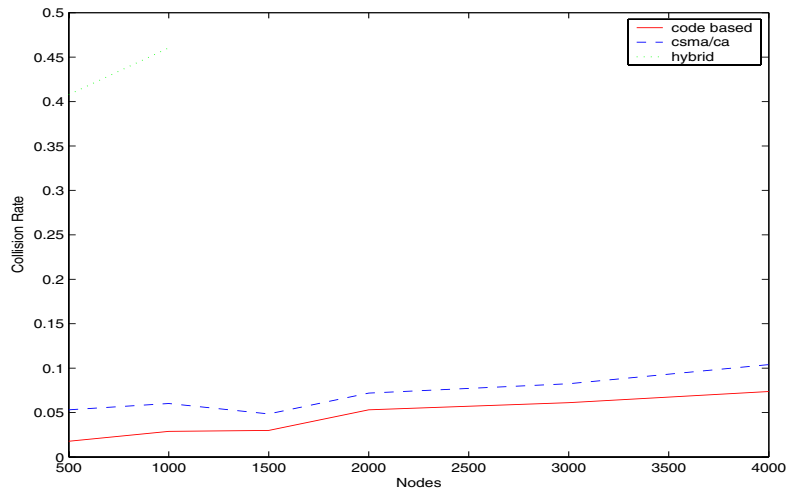

Figure 10. The collision rate as the size of the network grows.

Figure 10 shows the collision rate for each protocol. Notice that both the code based and csma/ca protocols have a significantly lower collision rate than the hybrid system. The code based protocol has a lower collision probability over the range of network sizes.

Figure 11 shows the average latency of routing level packets for each protocol. The code based and csma/ca pro-

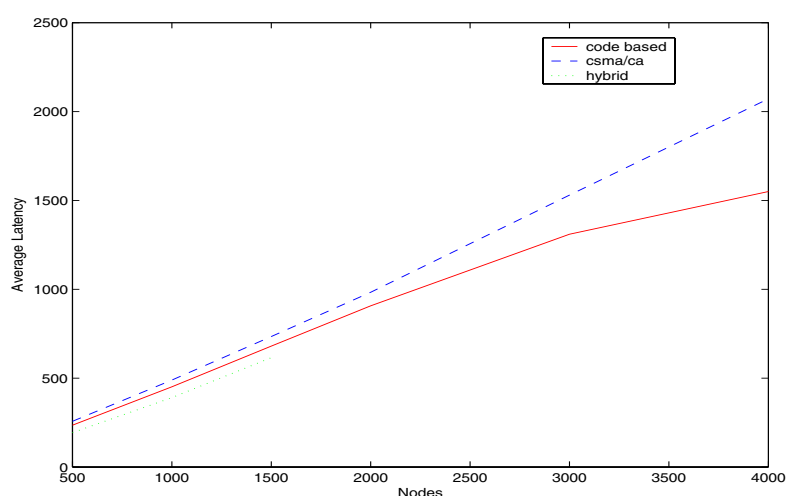

Figure 11. Average latency of routing layer packets as the size of the network grows.

tocols perform almost identically until about 3000 nodes are reached, where the code based protocol starts to perform significantly better than csma/ca.

Overall the code based protocol seems to outperform the csma/ca based and hybrid protocols as the size of the network grows.

\subsection{Network traffic scaling}

We fixed the number of nodes in the system at 1000 and varied the rate at which traffic was generated by varying $\lambda$.

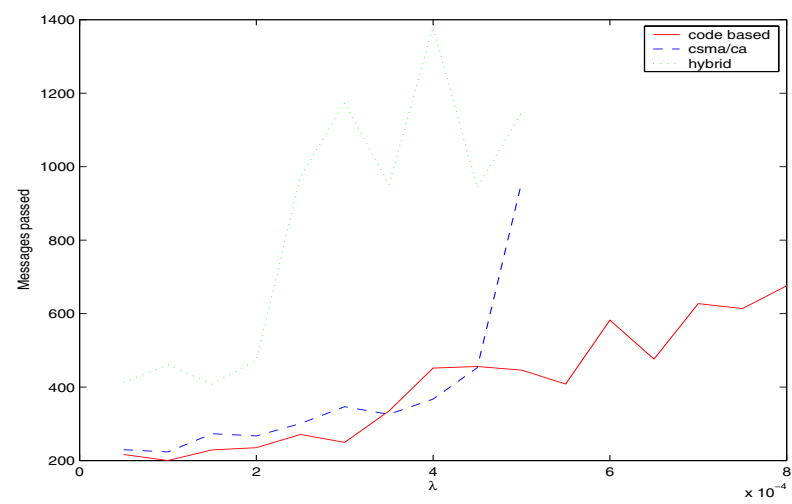

Figure 12. The number of routing layer messages passed as more traffic is generated.

Figure 12 shows the number of messages passed for each protocol as network traffic increases. As we would expect as more traffic is generated more messages are passed overall. The hybrid protocol seems to pass the most messages with the csma/ca and code based protocols performing similarly.

Figure 13 shows collision rate for each protocol as network traffic increases. The hybrid protocol reaches satura- 


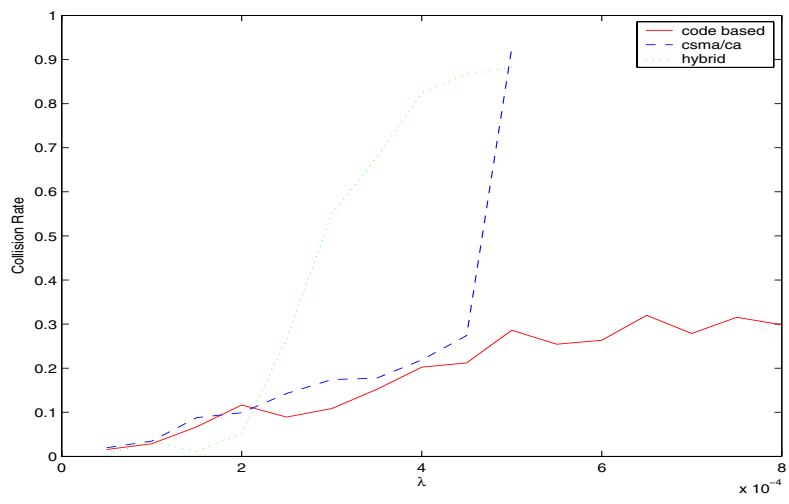

Figure 13. The collision rate as more traffic is added to the system.

tion with the lowest traffic rate of the three protocols. The csma/ca protocol also reaches saturation soon after, but the code based system seems to taper off with a collision rate around $30 \%$.

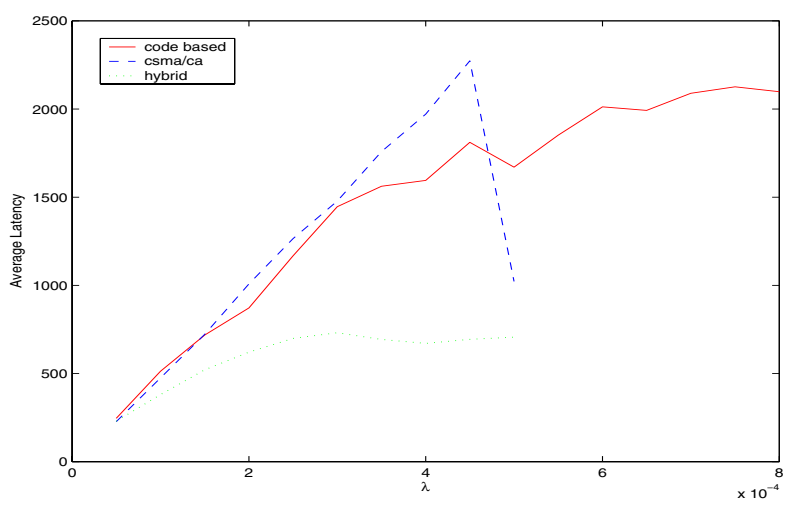

Figure 14. The average routing layer packet latency as more traffic is generated.

Figure 14 shows average routing layer packet latency for each protocol as network traffic increases. As expected, in general when we pump more traffic into the system the average latency increases. The hybrid protocol seems to perform best of the 3 protocols with the csma/ca and code based protocols performing somewhat similarly, until the csma/ca protocol is near saturation.

Although the other protocols may seem to have favorable throughput numbers it seems the code based system is better because it does not saturate as quickly as the network traffic is increased.

Throughout the situations tested as network characteristics scale up the code based system, using approximate superimposed codes, outperforms the other two methods.

\section{Summary and Conclusions}

We examined several coding constructs for use in multihop wireless networks. Of the currently used codes the incidence matrix constructed superimposed codes had better growth rates when scaling to large numbers of nodes, and offered some flexibility in terms of code density. There appear to be great gains possible if we are willing to relax the disjunct property of superimposed codes and use almost disjunct codes.

We also examined how various properties of the codes used would effect localized network performance. As we increased the number of nodes in the system a very low density code appears to be desirable. Increasing the reception probability locally would also have a two-fold effect, it not only would increase the network performance in terms of latency and throughput, but will also reduce the power costs of the system by avoiding collisions.

In our simulations there was not a clear winner in terms of throughput or latency, but in terms of collision rate it appeared the almost disjunct code performed the best. The hybrid approach showed some potential, but also showed its limitations when scaling the number of nodes in the system.

\section{References}

[1] A. J. Macula, V. V. RYkov, S. Y. Trivial two-stage group testing for complexes using almost disjunct matrices. Discrete and Applied Mathematics 137 (2004), 97-107.

[2] Chlamtac, I., And Faragó, A. Making transmission schedules immune to topology changes in multihop packet radio networks. IEEE/ACM Transactions on Networking 2, 1 (February 1994), 23-29.

[3] JU, J.-H., AND LI, V. O. K. An optimal topologytransparent scheduling method in multihop packet radio networks. IEEE/ACM Transactions on Networking 6, 3 (June 1998), 298-306.

[4] Kautz, W. H., And Singleton, R. Nonrandom binary superimposed codes. IEEE Transaction on Information Theory 10 (October 1964), 363-377.

[5] Macula, A. J. Simple construction of d-disjunct matrices with certain constant weights. Discrete Mathematics 162 (1996), 311-312.

[6] Syrotiuk, V. R., Colbourn, C. J., And Ling, A. C. H. Topology-transparent scheduling for manets using orthogonal arrays. Preprint, May 2003.

[7] The MathWorks, I. Matlab. http://www.mathworks.com/index.shtml. 